\title{
Desmame da ventilação mecânica em pacientes com COVID-19*
}

\author{
Weaning from mechanical ventilation in patients with COVID-19*
}

\begin{abstract}
CASTRO, Larissa Araújo de ${ }^{1}$; ROCHA, Ângelo Roncalli Miranda ${ }^{2}$; CAMILLO, Carlos Augusto $^{3}$; em nome do Comitê COVID-19 da ASSOBRAFIR.
\end{abstract}

\begin{abstract}
Resumo
O fisioterapeuta é fundamental no tratamento de pacientes com COVID-19, em especial na condução da ventilação mecânica $(\mathrm{VM})$, desde a estratégia inicial até o reestabelecimento da ventilação espontânea. O corpo de evidências a respeito do desmame da VM em pacientes com COVID-19 ainda é incipiente e, portanto, sua execução deve combinar recomendações internacionalmente aceitas sobre desmame com medidas adicionais de segurança. Recomenda-se a avaliação diária da possibilidade de desmame, observando critérios como a capacidade de oxigenação e proteção de vias aéreas. Índices preditivos podem auxiliar, porém, devemos evitar aqueles que envolvem desconexão do ventilador mecânico pelo risco de aerossolização. Pela mesma razão, recomenda-se que o teste de respiração espontânea seja realizado em ventilação com suporte pressórico. A VM não invasiva pós extubação permanece indicada quando houver estrutura adequada para sua realização. Aos pacientes traqueostomizados em respiração espontânea, recomenda-se o uso de filtro trocador de calor e umidade de alta eficiência.
\end{abstract}

Palavras-chave: Fisioterapia; Desmame; COVID-19.

* Revisado por membros do Comitê COVID-19 da ASSOBRAFIR, nomeado por meio do memorando N 003/2020. Esta publicação é uma atualização da Comunicação oficial "Desmame da ventilação mecânica em pacientes com COVID-19", chancelada pelo Comitê COVID-19 da ASSOBRAFIR, originalmente escrita pelos mesmos autores e divulgada em 24/06/2020 no endereço eletrônico https://assobrafir.com.br/covid-19/.

1 Departamento de Fisioterapia, Universidade Estadual de Londrina (UEL), Londrina, Paraná, Brasil.

Email: laridecastro13@gmail.com. LAC - https://orcid.org/0000-0002-9034-8662

${ }^{2}$ Hospital Escola Dr. Helvio Auto, Universidade de Ciências da Saúde de Alagoas, Maceió, Alagoas, Brasil.

CAC - https://orcid.org/0000-0001-7648-8573

3 Departamento de Fisioterapia, Universidade Estadual de Londrina (UEL), Londrina, Paraná, Brasil. Departamento de Ciências da Reabilitação, Centro de Pesquisa - Campus Piza, Universidade Pitágoras UNOPAR, Londrina, Paraná, Brasil. ARMR - https://orcid.org/0000-0002-7944-7168 


\section{Abstract}

The physiotherapist is essential for the treatment of patients with COVID-19, especially in the management of mechanical ventilation (MV), from the initial MV settings to the reestablishment of spontaneous breathing. The body of evidence regarding weaning from MV in patients with COVID-19 is still incipient and, therefore, its implementation should combine internationally accepted weaning recommendations with additional safety measures. Daily screenings of potentially eligible patients for weaning are recommended, observing criteria such as oxygenation and patient's capacity of protecting the airways. Predictive indexes can be helpful; however, those involving disconnection from the mechanical ventilator must be avoided due to risk of aerosolization. For the same reason, it is recommended that spontaneous breathing trials should be performed using MV with pressure support. Non-invasive mechanical ventilation after extubation remains indicated when an adequate environment is guaranteed. For tracheostomized patients in spontaneous breathing, the use of a highly efficient heat and moisture exchanger filter is recommended.

Keywords: Physiotherapy; Weaning; COVID-19.

\section{Objetivo}

O objetivo do presente posicionamento é fornecer subsídios para a atuação da fisioterapia em pacientes com COVID-19 que necessitam de terapia intensiva, com o foco na condução do desmame da ventilação mecânica, para que este seja realizado de forma efetiva e com a maior segurança possível.

\section{Contextualização}

A Organização Mundial da Saúde (OMS) classifica a gravidade da doença de pacientes com COVID-19 de acordo com o número de síndromes clínicas associadas a infecção do vírus. Estas síndromes podem ser classificadas como: 1- Doença Leve; 2- Pneumonia; 3- Pneumonia Grave; 4Síndrome do desconforto respiratório agudo (SDRA); 5- Sepse e; 6- Choque séptico ${ }^{1}$. Pacientes a partir do grupo 4 (i.e., com SDRA) necessitarão de suporte ventilatório invasivo e serão submetidos a processo de desmame ventilatório após a conclusão da doença. O posicionamento da ASSOBRAFIR sobre o desmame desses pacientes segue recomendações internacionais de pacientes em SDRA, uma vez que não há até a presente data recomendações específicas para o desmame de pacientes com COVID-19.

\section{Indicação de Desmame}

Pacientes que evoluem com as formas mais graves da doença podem permanecer hipoxêmicos por um longo período, necessitando de parâmetros elevados de ventilação mecânica e, por vezes, diversas sessões de pronação ${ }^{2}$. Ainda que, por essas razões, o desmame da ventilação mecânica esteja sendo pensado com cautela, a OMS recomenda que sejam utilizados protocolos de desmame nos quais a possibilidade de respiração espontânea seja avaliada diariamente ${ }^{1}$.

O processo de desmame deve, portanto, ser considerado diariamente nas seguintes condições:

- Nível de consciência adequado (Escala de Coma de Glasgow $\geq 8$ );

- Oxigenação adequada: $\mathrm{PaO}_{2} \geq 60 \mathrm{mmHg}$ com $\mathrm{FiO}_{2} \leq 0,40$ e $\mathrm{PEEP}<10^{3}$;

- Estabilidade hemodinâmica: pressão arterial média $\geq 60 \mathrm{mmHg}$ sem necessidade de vasopressores (ou em doses baixas); 
- Ausência de secreções excessivas (e.g., mais do que 1 aspiração a cada 2 horas);

- Capacidade de proteção de vias aéreas: pico de fluxo expiratório $>601 / \mathrm{min}$; $^{*}$

- Não apresentar suspeita de edema de vias aéreas. ${ }^{* *}$

${ }^{*}$ Nota: A tosse deve ser avaliada com o paciente conectado ao ventilador. Apesar de pouca evidência disponível, pode-se utilizar a medida do pico de fluxo expiratório no ventilador após a solicitação de tosse pelo paciente. Valores $>601 /$ min estão relacionados com sucesso do desmame. ${ }^{4}$

${ }^{*}$ Nota: A suspeita de edema de vias aéreas pode ser levantada pela equipe caso o procedimento de intubação tenha sido difícil ou traumático. Dada a baixa sensibilidade do teste e o risco de aerossolização, não podemos emitir recomendação para fazer ou não o teste de vazamento de cuff. ${ }^{5}$

Além disso, há vários preditores de sucesso de desmame que podem ajudar o intensivista na decisão de uma provável extubação ${ }^{6}$. Um preditor simples e, portanto, muito utilizado é o índice de respiração rápida superficial, sendo o ponto de corte recomendado $<80 .{ }^{7}$ Porém, é importante levar em consideração que a medida pode variar para diferentes níveis de pressão de suporte ou mesmo na presença do fluxo de base $e^{8}$. Outrossim, os disparos ineficazes não são contabilizados pelo ventilador mecânico na mensuração do IRRS, podendo falsear a medida. Em virtude disto, recomendamos não utilizar o IRRS como variável principal na avaliação do desmame.

Medidas de PImax são de grande valia na avaliação do risco de falha do desmame, porém, o procedimento envolve a desconexão do paciente do ventilador e deve, sempre que possível, ser evitado. Não há evidência suficiente para realizar a avaliação da força muscular respiratória através da função Maximal Inspiratory Pressure (MIP) ou Negative Inspiratory Force (NIF) disponível em alguns ventiladores mecânicos. Dessa forma, não recomendamos essas medidas em substituição à tradicional manuvacuometria com válvula unidirecional.

Caso o paciente se enquadre nos critérios descritos acima a equipe deve considerar a possibilidade de prosseguir com o desmame.

\section{Execução do desmame}

O teste de respiração espontânea deve ser utilizado para avaliar a capacidade do paciente em sustentar um padrão ventilatório adequado após a extubação. Entretanto, não é indicado fazer o teste desconectando-se o paciente do ventilador mecânico, como por exemplo, no "tubo T". O TRE deve ser realizado, preferencialmente ${ }^{9,10}$ :

- Em ventilação com suporte pressórico (VSP) de 5 a $7 \mathrm{cmH}_{2} \mathrm{O}$ durante 30 minutos ${ }^{11-13}$;

- Se houver dúvida, realize o TRE de forma mais criteriosa, utilizando-se o menor suporte pressórico $\left(5 \mathrm{cmH}_{2} \mathrm{O}\right)$;

- Avalie continuamente sinais de intolerância: esforço respiratório, $\mathrm{FR}>30 \mathrm{rpm}, \mathrm{SpO}_{2}<90 \%$, FC $>140 \mathrm{bpm}, \mathrm{PAS}>180 \mathrm{ou}<90 \mathrm{mmHg}$, agitação, sudorese e alteração do nível de consciência.

No caso de sucesso no TRE, a extubação está indicada. 


\section{Falha do desmame}

Caso o paciente apresente falha no desmame, deve-se avaliar o motivo que levou a falha (e.g., avaliar quadro geral, neurológico, estabilidade hemodinâmica, sinais de esforço ventilatório, etc.) e considerar novas avaliações caso ainda tenham sido realizadas. Recomenda-se aguardar $24 \mathrm{~h}$ antes de uma nova tentativa ${ }^{9,10}$.

\section{Extubação}

É importante salientar que o modo de extubação deve ser discutido com a equipe do serviço seguindo-se um protocolo rigoroso que assegure a segurança do intensivista e do paciente. Antes da extubação é necessário preparar a sala e deixar todos os materiais que serão utilizados a disposição. Faça um checklist com a lista de materiais necessários, isso irá garantir que não haja necessidade de sair do ambiente durante o procedimento. Idealmente, a extubação deve ser feita em duplas para diminuir risco de falha e a duração do procedimento. Por fim, monitore sinais de falha da extubação e ajuste o fluxo de oxigênio da interface ventilatória para manter uma oxigenação adequada.

Nota: A utilização de máscaras ou sistemas de alto-fluxo pós extubação e/ou qualquer procedimento que produza névoa ou aerossol ainda não possuem ampla recomendação.

Nota: Minimize o risco de tosse ou de exposição a secreções. Evite procedimentos que irritem as vias aéreas e estimulem a tosse.

Nota: O uso de dispositivos ventilatórios supra-glóticos (supraglotic airway devices) devem ser evitados por estimularem a tosse.

O fluxograma a seguir é uma sugestão aos serviços para realização das etapas de extubação do paciente com COVID-19 (Figura 1). ${ }^{14}$

\section{Suporte ventilatório após a extubação}

A suplementação de oxigênio após a extubação deve ser realizada, quando houver necessidade, para a manutenção da $\mathrm{SpO}_{2}$ entre 90 e $96 \% .{ }^{15}$ Os recursos mais recomendados até então são a cânula nasal de oxigênio com fluxo até $6 \mathrm{~L} / \mathrm{min}$ ou a máscara não reinalante até $10 \mathrm{~L} / \mathrm{min}$. Em caso de insuficiência respiratória, a reintubação não deve ser protelada.

A ventilação não invasiva (VNI) tem sido um recurso terapêutico de grande utilidade para evitar falha após extubação, com indicações já bem estabelecidas na literatura. ${ }^{9} 10$ Porém, no caso dos pacientes com COVID-19, o emprego deste recurso ainda está sendo bastante debatido, pois pode haver grande dispersão do vírus em aerossol e, desta maneira, maior risco de contaminação da equipe de saúde ${ }^{16}$. Até o momento, sua indicação fica limitada a casos específicos a serem discutidos com a equipe multiprofissional e a serviços que disponham de estrutura adequada para realização da VNI. Por estrutura adequada entende-se: isolamentos com pressão negativa, interfaces para realização de ventilação não invasiva com boa vedação e sem válvula de exalação, circuitos de ramo duplo, trocador de calor e umidade proximal associado a um filtro de barreira (HEPA) na parte distal do ramo expiratório ou filtro trocador de calor e umidade (HMEF de alta eficiência) proximal. 
Figura 1 | Fluxograma para extubação do paciente com COVID-19.

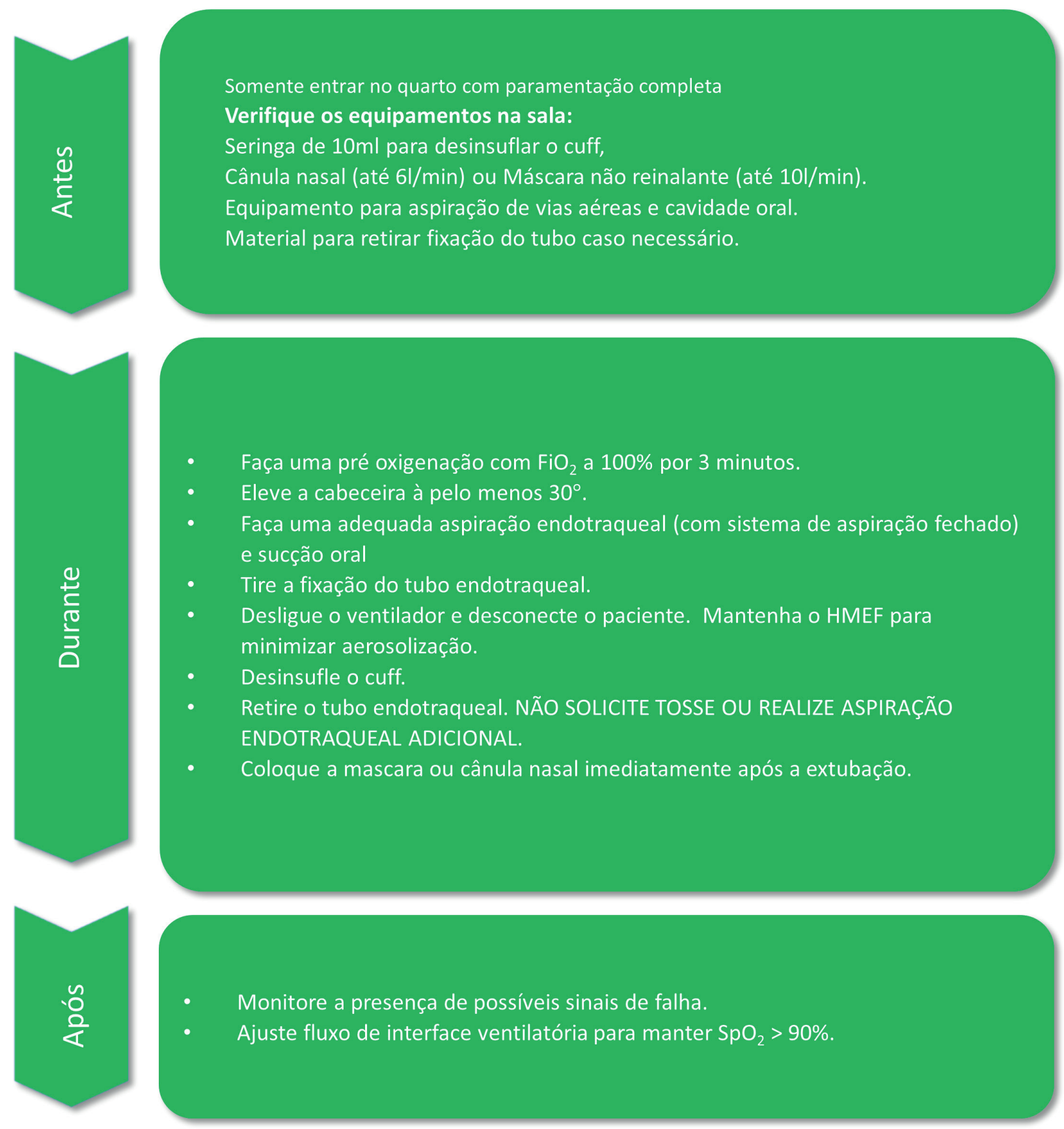

\section{Considerações ao paciente traqueostomizado ${ }^{17}$}

Uma boa parte dos pacientes com COVID-19 evoluem em ventilação mecânica prolongada e acabam por necessitar de traqueostomização. Muito ainda se tem discutido sobre a melhor forma de conduzir a respiração espontânea desses pacientes. Idealmente, um HMEF (ou filtro com eficiência mínima de $99,5 \%$ para partículas menores de $0,3 \mu \mathrm{m}^{18}$ ) deve ser utilizado acoplado ao sistema de aspiração fechado (Figura 2). O mesmo se aplica para filtros específicos para traqueostomia (i.e., o filtro deve ser misto e possuir alta eficiência de filtragem conforme descrito acima). A suplementação 
de oxigênio (quando necessária) deve ser feita em baixo fluxo e sem a utilização adicional de água, isto é, a umidificação fica totalmente por conta do HMEF. Nestas situações, deve-se ter cautela com a possibilidade do aumento da resistência que esse dispositivo pode promover e, consequentemente, aumentar o trabalho da respiração para o paciente. Caso haja sinais de desconforto respiratório por aumento da resistência proporcionado pelo HMEF, sugerimos descontinuar a utilização do mesmo. O HMEF deve ser utilizado com cautela também no caso de pacientes hipersecretivos. Nestes casos, o serviço deve avaliar se há possibilidade de realizar a troca mais frequente do dispositivo ou se o paciente já pode permanecer sem filtro de barreira, uma vez que o uso de HMEF não é indicado em casos de hipersecreção pulmonar.

Os demais cuidados são os costumeiramente dispensados ao paciente traqueostomizado. Vale ressaltar apenas que atenção especial deve ser dada as pressões de cuff, mantendo o balonete insuflado entre 20 e $30 \mathrm{cmH}_{2} \mathrm{O}$ para minimizar a aerolização. Por fim, a troca do tubo de traqueotomia deve ser adiada até que o paciente não seja mais infeccioso de acordo com a equipe de Doenças Infecciosas.

Figura 2 | Exemplo de dispositivo para pacientes traqueostomizados com COVID-19. 1) Sistema de aspiração fechado; 2) Filtro misto de alta eficiência; 3) Entrada de suplementação de $\mathrm{O}_{2}$ (caso necessário).

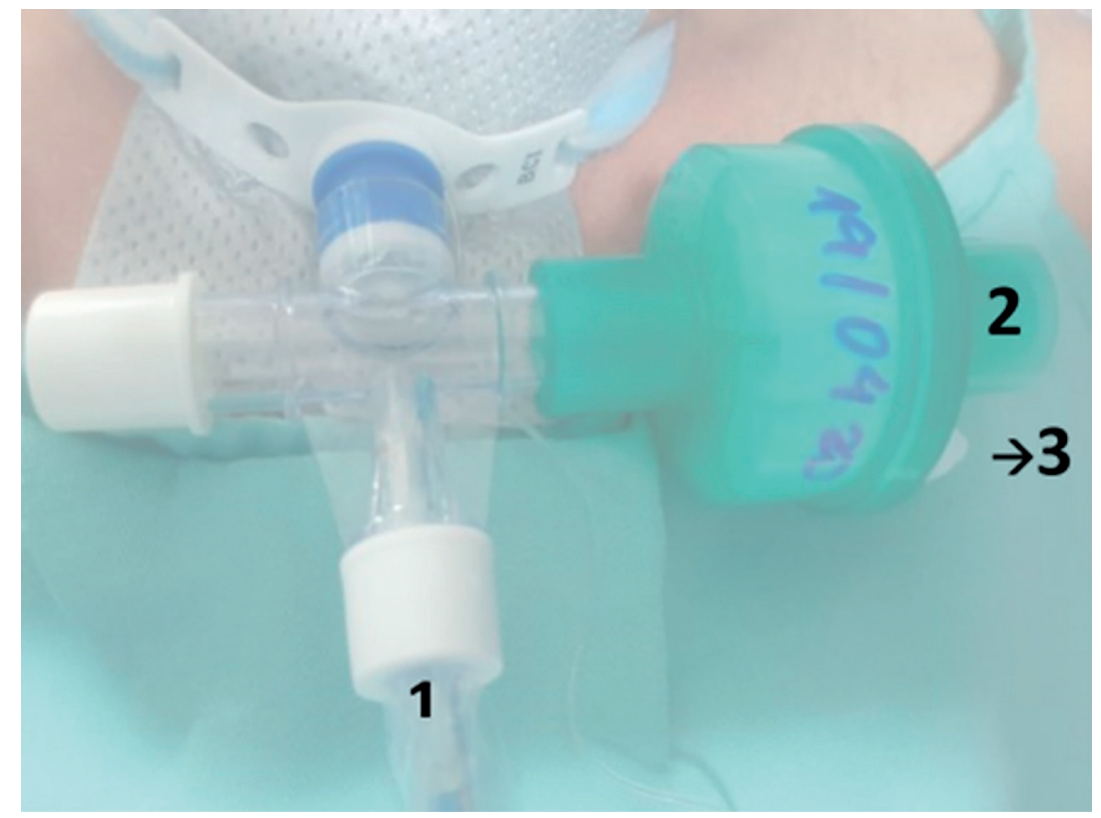

Fonte: Foto gentilmente cedida por Jorge Dantas. 


\section{Considerações Finais}

Os direcionamentos apresentados levam em consideração o importante papel do fisioterapeuta no tratamento de pacientes com COVID-19, em especial na condução da ventilação mecânica. No que diz respeito ao desmame da ventilação mecânica, evidências científicas específicas para pacientes com COVID-19 ainda são escassas e, portanto, sua execução deve combinar recomendações internacionalmente aceitas sobre desmame com medidas adicionais de segurança para proteção da equipe de saúde. Nesse sentido, esperamos que as presentes orientações auxiliem o fisioterapeuta a manejar o desmame da ventilação mecânica de pacientes com COVID-19 da forma mais efetiva e segura possível, baseando-se na melhor evidência disponível até o momento.

\section{Referências}

1. WHO/OMS. Clinical management of severe acute respiratory infection (SARI) when COVID-19 disease is suspected: Interim guidance, 13 March 2020 [Internet]. Geneva: World Health Organization; 2020. Available from: https://www.who.int/docs/default-source/coronaviruse/clinical-managementof-novel-cov.pdf

2. Rosenbaum L. Facing Covid-19 in Italy - Ethics, Logistics, and Therapeutics on the Epidemic's Front Line. N Engl J Med. 2020 May 14;382(20):1873-1875. doi: 10.1056/NEJMp2005492. Epub 2020 Mar 18.

3. Writing Group for the Alveolar Recruitment for Acute Respiratory Distress Syndrome Trial I, Cavalcanti AB, Suzumura EA, Laranjeira LN, Paisani DM, Damiani LP, et al. Effect of Lung Recruitment and Titrated Positive End-Expiratory Pressure (PEEP) vs Low PEEP on Mortality in Patients With Acute Respiratory Distress Syndrome: A Randomized Clinical Trial. JAMA. 2017 Oct 10;318(14):1335-1345. doi: 10.1001/jama.2017.14171.

4. Gobert F, Yonis H, Tapponnier R, Fernandez R, Labaune M-A, Burle J-F, et al. Predicting Extubation Outcome by Cough Peak Flow Measured Using a Built-in Ventilator Flow Meter. Respir Care. 2017 Dec;62(12):1505-19. doi: 10.4187/respcare.05460. Epub 2017 Sep 12.

5. Schnell D, Planquette B, Berger A, Merceron S, Mayaux J, Strasbach L, et al. Cuff Leak Test for the Diagnosis of Post-Extubation Stridor: A Multicenter Evaluation Study. J Intensive Care Med. 2019 May;34(5):391-6. doi: 10.1177/0885066617700095. Epub 2017 Mar 27.

6. Nemer SN, Barbas CS. Predictive parameters for weaning from mechanical ventilation. J Bras Pneumol. 2011 Sep-Oct;37(5):669-79. doi: 10.1590/s1806-37132011000500016.

7. Souza LC, Lugon JR. The rapid shallow breathing index as a predictor of successful mechanical ventilation weaning: clinical utility when calculated from ventilator data. J Bras Pneumol. 2015 NovDec;41(6):530-5. doi: 10.1590/S1806-37132015000000077.

8. El-Khatib MF, Zeineldine SM, Jamaleddine GW. Effect of pressure support ventilation and positive end expiratory pressure on the rapid shallow breathing index in intensive care unit patients. Intensive Care Med. 2008 Mar;34(3):505-10. doi: 10.1007/s00134-007-0939-x. Epub 2007 Dec 1.

9. Barbas CS, Isola AM, Farias AM, Cavalcanti AB, Gama AM, Duarte AC, et al. Brazilian recommendations of mechanical ventilation 2013. Part I. Rev Bras Ter Intensiva. 2014 AprJun;26(2):89-121. doi: 10.5935/0103-507x.20140017. 
10. Barbas CS, Ísola AM, Farias AM, Cavalcanti AB, Gama AM, Duarte AC, et al. Brazilian recommendations of mechanical ventilation 2013. Part 2. Rev Bras Ter Intensiva. 2014 JulSep;26(3):215-39. doi: 10.5935/0103-507x.20140034.

11. Subirà C, Hernández G, Vázquez A, Rodríguez-García R, González-Castro A, García C, et al. Effect of Pressure Support vs T-Piece Ventilation Strategies During Spontaneous Breathing Trials on Successful Extubation Among Patients Receiving Mechanical Ventilation: A Randomized Clinical Trial. JAMA. 2019 Jun 11;321(22):2175-2182. doi: 10.1001/jama.2019.7234.

12. Zhang B, Qin YZ. Comparison of pressure support ventilation and T-piece in determining rapid shallow breathing index in spontaneous breathing trials. Am J Med Sci. 2014 Oct;348(4):300-5. doi: 10.1097/MAJ.0000000000000286.

13. Li Y, Li H, Zhang D. Comparison of T-piece and pressure support ventilation as spontaneous breathing trials in critically ill patients: a systematic review and meta-analysis. Crit Care. 2020 Feb 26;24(1):67. doi: 10.1186/s13054-020-2764-3.

14. Cook TM, El-Boghdadly K, McGuire B, McNarry AF, Patel A, Higgs A. Consensus guidelines for managing the airway in patients with COVID-19: Guidelines from the Difficult Airway Society, the Association of Anaesthetists the Intensive Care Society, the Faculty of Intensive Care Medicine and the Royal College of Anaesthetists. Anaesthesia. 2020 Jun;75(6):785-799. doi: 10.1111/anae.15054. Epub 2020 Apr 1.

15. Alhazzani W, Møller MH, Arabi YM, Loeb M, Gong MN, Fan E, et al. Surviving Sepsis Campaign: Guidelines on the Management of Critically Ill Adults with Coronavirus Disease 2019 (COVID-19). Crit Care Med. 2020 Jun;48(6):e440-e469. doi: 10.1097/CCM.0000000000004363.

16. Tran K, Cimon K, Severn M, Pessoa-Silva CL, Conly J. Aerosol generating procedures and risk of transmission of acute respiratory infections to healthcare workers: a systematic review. PLoS One. 2012;7(4):e35797. doi: 10.1371/journal.pone.0035797. Epub 2012 Apr 26.

17. Heyd CP, Desiato VM, Nguyen SA, O'Rourke AK, Clemmens CS, Awad MI, et al. Tracheostomy protocols during COVID-19 pandemic. Head Neck. 2020 Jun;42(6):1297-1302. doi: 10.1002/ hed.26192. Epub 2020 May 2.

18. Baron P. Generation and Behavior of Airborne Particles (Aerosols). Atlanta: National Institute for Occupational Safety and Health, Centers for Disease Control and Prevention; [2020]. Available from: https://www.cdc.gov/niosh/topics/aerosols/pdfs/Aerosol 101.pdf

Submissão em: 07/06/2020

Aceite em: 15/07/2020 